\title{
The effect of growth differentiation factor-5, 6, 7 in chondrogenic cell differentiation of ATDC-5
}

\author{
Yuji Hatakeyama ${ }^{1,}$ *, Yuko Matsuda ${ }^{2}$, Junko Hatakeyama ${ }^{3}$, Kyoko Oka ${ }^{4}$, Hisashi Anan ${ }^{3}$, \\ Eichi Tsuruga $^{5}$, Tetsuichiro Inai ${ }^{1}$, Hiroyuki Ishikawa ${ }^{2}$, Yoshihiko Sawa ${ }^{1}$ \\ ${ }^{1}$ Section of Functional Structure, Department of Morphological Biology, Fukuoka Dental College, Fukuoka, Japan \\ ${ }^{2}$ Section of Orthodontics, Department of Oral Growth and Development, Fukuoka Dental College, Fukuoka, Japan \\ ${ }^{3}$ Section of Operative Dentistry, Department of Odontology, Fukuoka Dental College, Fukuoka, Japan \\ ${ }^{4}$ Section of Pediatric Dentistry, Department of Oral Growth and Development, Fukuoka Dental College, Fukuoka, Japan \\ ${ }^{5}$ Department of Pathologic Aanalysis, Division of Medical Sciences, Hirosaki University Graduate School of Health Sciences, Hirosaki, \\ Japan
}

\section{Email address:}

hatakeyy@college.fdcnet.ac.jp (Y. Hatakeyama)

\section{To cite this article:}

Yuji Hatakeyama, Yuko Matsuda, Junko Hatakeyama, Kyoko Oka, Hisashi Anan, Eichi Tsuruga, Tetsuichiro Inai, Hiroyuki Ishikawa, Yoshihiko Sawa. The Effect of Growth Differentiation Factor-5, 6, 7 in Chondrogenic Cell Differentiation of ATDC-5. American Journal of BioScience. Vol. 2, No. 5, 2014, pp. 182-186. doi: 10.11648/j.ajbio.20140205.13

\begin{abstract}
The proteins known as growth differentiation factors (GDFs) are members of the BMP family; GDF-5, -6, and -7 play important roles in skeletogenesis, especially with regard to chondrogenesis. The functional differences among these GDFs in chondrogenesis and chondrocyte cell differentiation remains unclear. Here, we attempt to assay cell proliferation, the production of chondrogenic matrices indicated by alcian blue intensity, and the profile of a chondrogenic cell differentiation marker gene in a cell culture of the chondrogenic cell line ATDC5 with or without recombinant mouse GDF-5, -6 , and -7 . After $24 \mathrm{~h}$ culture, the ATDC5 cell number was significantly decreased in the exogenous presence of each GDF compared with a control culture absent all GDFs. On the other hand, alcian blue staining of cell cultures after $2 \mathrm{w}$ culture showed significantly increased intensity compared with that of the control. The expression levels of the chondrogenic cell differentiation marker genes Sox 9 and aggrecan were increased after $24 \mathrm{~h}$ and $48 \mathrm{~h}$ culture by all GDFs, but were significantly increased in the presence of GDF-5 compared with the presence of GDF-6 or -7. These findings suggest that GDF-5, -6, and -7 could all promote chondrogenic cell differentiation of ATDC5, but GDF-5 may induce chondrogenic genes more potently than GDF-6 and -7 do.
\end{abstract}

Keywords: Growth Differentiation Factors (GDFs), Chondrogenesis, ATDC-5

\section{Introduction}

The proteins known as growth differentiation factors (GDFs) are members of the BMP family, which in turn belongs to the transforming growth factor beta (TGF- $\beta$ ) superfamily. Based on the similarity of their amino acid sequences, GDF-5, GDF-6 and GDF-7 can be classified as a subgroup of GDFs. Mutation of GDF-5 has been identified as associated with skeletal growth and development in mice [1] and humans [2]. GDF-5 has chondro- and osteo-inductive functions both in vivo [3] and in vitro [4]. Both GDF-5 and GDF-6 are present in adult bovine and human articular cartilage; in vitro, they stimulate the metabolic activity of chondrocytes [5]. In human mesenchymal stem cells, GDF-6 promotes proteoglycan synthesis and chondrogenic gene expression [6]. In a mouse pluripotent mesenchymal cell line, GDF-7 enhanced alcian blue staining, which is indicative of extracellular matrix formation [7]. Experiments with GDF-7 deficient mice have revealed that, in the absence of GDF-7, the rate of endochondral bone growth is affected through modulation of the hypertrophic zone in growth plate chondrocytes [8]. Furthermore, in chondrocyte-derived bovine articular cartilage cultured on a PG scaffold, GDF-6 and GDF-7 increased collagen and glycosaminoglycans, which serve as extracellular matrices of cartilage [9]. In spite of this accumulated knowledge, however, the functional differences among GDF-5, GDF-6 and GDF-7 from the same cell source have not yet been identified. In this study, we attempt to clarify the 
differences among these growth factors, with particular attention to their roles in chondrogenic cell differentiation in a mouse chondrogenic cell line.

\section{Materials and Methods}

\subsection{Cell Culture}

Cells from the mouse chondrogenic cell line ATDC5 were obtained from Riken Bioresource Center Cell Bank (Tsukuba, Japan). ATDC5 cells were cultured to reach an appropriate cell number for each experiment using cell maintenance medium consisting of a 1:1 mixture of Dulbecco's Modified Eagle Medium (D-MEM) and Ham's F12 (Invitrogen/Life Technologies, Tokyo, Japan), 5\% fetal bovine serum (FBS, Equitech-Bio Inc., Tokyo, Japan), $3 \times$ $10^{-8} \mathrm{M}$ sodium selenite (Wako, Osaka, Japan), $10 \mu \mathrm{g} / \mathrm{ml}$ holo-form bovine transferrin (Invitrogen), and antibiotics (100U/ml of penicillin-G and $100 \mathrm{mg} / \mathrm{ml}$ of streptomycin) at $37^{\circ} \mathrm{C}$ in a humidified atmosphere of $5 \% \mathrm{CO} 2$ in air. In each experiment, cells were treated with various concentrations of recombinant mouse GDF-5, -6 , and -7 (R\&D Systems, Minneapolis, MN, USA) diluted in $0.1 \%$ bovine serum albumin (Sigma Aldrich, Tokyo, Japan) in Hank's balanced salt solution and cultured for the indicated time.

\subsection{Cell Proliferation}

The proportional numbers of cells were counted as described previously [10] using a Cell Counting Kit-8 (Dojindo Laboratories, Kumamoto, Japan), which de-pends on the measurement of a highly water-soluble formazan dye produced from tetrazolium salts. ATDC5 cells were seeded at $1.0 \times 10^{3}$ cells per well on a 96-well plate, and after initial cell attachment the medium was changed that included $12.5,25,50$, or $100 \mathrm{ng} / \mathrm{ml}$ of GDF-5, -6 , or -7 . After 24 hours of cell culture, cells were incubated with counting reagent for one hour according to the manufacturer's instructions. The relative cell number was then determined by measuring light absorbance at a wavelength of 450nm (1420 Multilabel counter ARVO MS; PerkinElmer, Waltham, MA, USA) using the formazan dye product in the cultures.

\subsection{Alcian Blue Staining}

The presence of sulfated proteoglycans, indicative of cartilaginous matrix production, was detected by alcian blue staining. After cell confluence, ATDC5 cells were seeded on 24-well plates and grown to the required cell number in a maintenance medium that included 12.5, 25, 50 or $100 \mathrm{ng} / \mathrm{ml}$ of GDF-5, -6 , or -7 . After two weeks of cell culture in this medium, which was changed twice per week, alcian blue staining was performed as described previously with minor modifications [4]. Briefly, cultures were fixed in $2 \%$ acetic acid in ethanol for $15 \mathrm{~min}$ at room temperature, rehydrated and stained overnight at room temperature with $0.5 \%$ Alcian Blue Stain Solution pH2.5 (Muto Pure Chemicals, Tokyo, Japan). The intensity of alcian blue staining was measured by absorbance at 600nm (1420 Multilabel counter ARVO MS, PerkinElmer) after the alcian blue stained cartilage matrices were solubilized with 4M guanidine hydrochloride.

\subsection{Reverse Transcription Polymerase Chain Reaction (RT-PCR)}

ATDC5 cells were cultured in the absence or presence of $50 \mathrm{ng} / \mathrm{ml}$ of GDF-5, -6 or -7 in maintenance medium. After 24 and 48 hours culture, total RNA was isolated from each culture's cells using an RNeasy Mini Kit (Qiagen, Inc., Valencia, CA, USA) according to the manufacturer's instructions. The expression levels of several chondrogenic differentiation marker genes (Sox9, Collagen Type II (Col2), Collagen Type X (ColX), and Aggrecan) were analyzed using real-time quantitative RT-PCR (MX3005P, Agilent Technologies, Tokyo, Japan); reverse transcription and real-time PCR were per-formed as previously reported with minor modifications [11]. Briefly, reverse transcription was performed using the iScript Reverse Transcription Supermix for RT-qPCR (Bio-Rad, Tokyo, Japan) according to the manufacturer's instructions. Real-time PCR was performed using iTaqTM SYBR Green Supermix with ROX (Bio-Rad) for 45 cycles, each consisting of $95^{\circ} \mathrm{C}$ for 30 seconds, $60^{\circ} \mathrm{C}$ for 45 seconds and $72^{\circ} \mathrm{C}$ for 30 seconds. Relative standard curves representing several 10-fold dilutions of standard cDNA (1:10:100:1000) were used for a linear regression analysis of unknown samples. The expression levels of each chondrogenic differentiation marker gene were assessed relative to that of glycealde-hyde-3-phosphate dehydrogenase (GAPDH). Amplimers designed for each gene have been previously reported [4].

\subsection{Statistical Analysis}

All data are expressed as the mean \pm standard deviation (SD) of the mean; sample numbers were 8 or 6 for each experiment. Statistical significance was determined using Krushkal-Wallis one-way analysis of variance (ANOVA) followed by a post-hoc t-test. Differences were considered significant at a $\mathrm{p}$ value of $<0.05$.

\section{Results}

\subsection{Cell Proliferation}

In order to compare the functions of GDFs in the regulation of cartilage formation systematically, we first analyzed their effects on cell proliferation. The proportional cell number of ATDC5 was determined by measurement of water-soluble formazan dye after 24 hours of culture in the presence of GDF-5, -6 , or -7 at $12.5,25,50$, or $100 \mathrm{ng} / \mathrm{ml}$ (Fig. 1). These concentrations were tested be-cause similar concentrations of GDF-5 have been used previously to elicit a cell proliferation response [4]. In the presence of GDF-5, the cell number was significantly and dose-dependently decreased at concentrations of 12.5, 25 and $100 \mathrm{ng} / \mathrm{ml}$ (Fig.1). In the presence of GDF-6, the cell 
number showed a tendency to decrease at all tested concentrations, although significant decreases were seen only between 12.5 and $100 \mathrm{ng} / \mathrm{ml}$ (Fig. 1). In the presence of GDF-7, the cell number was significantly decreased at concentrations of 12.5, 50 and 100ng/ml (Fig. 1).

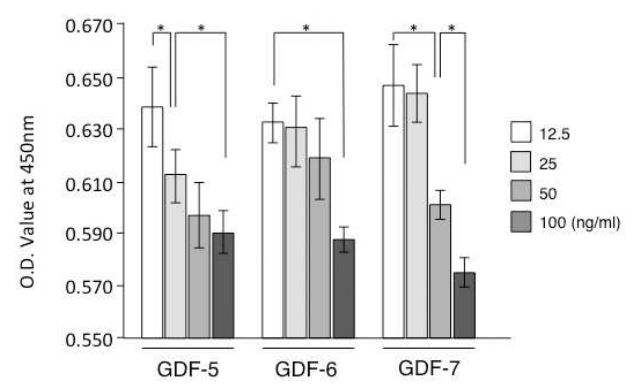

Figure 1. Proportional cell number of ATDC5 in the presence each dose of GDFs after $24 \mathrm{~h}$ culture. ${ }^{*} p<0.05$ when compared with each dose of GDFs.

\subsection{Alcian Blue Staining}

Next we investigated the production of cartilaginous matrices by ATDC5 cells, which can be measured with alcian blue staining after two weeks of culture. Cultures of ATDC5 were stained alcian blue at each concentration of each GDF (Fig. 2A), and the intensity of alcian blue staining increased in a dose-dependent manner (Fig. 2B).

\subsection{Gene Expression}

We examined the expression levels of cartilage marker transcripts, such as Sox9, Col2, ColX, and aggrecan, using RT-PCR. In the absence of GDFs in the maintenance medium, Sox9, Col2 and ColX did not in decrease in expression in ATDC5 cells after 24 and 48 hours of culture (Fig. 3A, B, C), though aggrecan did (Fig. 3D). In the presence of $50 \mathrm{ng} / \mathrm{ml}$ of GDF-5, -6 or -7 , on the other hand, Sox9, ColX and aggrecan showed increased expression in ATDC5 cells after 24 and 48 hours (Fig. 3A, B, C and D). Furthermore, the presence of GDF-5 significantly increased the gene expression of Sox 9 after 24 hours of culture (Fig. $3 \mathrm{~A}$ ) and that of aggrecan after 48 hours of culture (Fig. 3D) compared with the presence of GDF- 6 or -7 after the equivalent culture period.
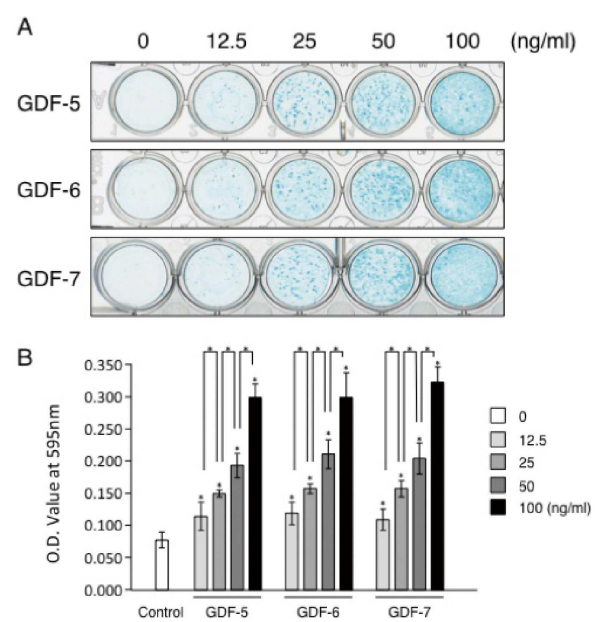

Figure 2. Alcian blue staining of ATDC5 cells and staining intensity assay of alcian blue treated by each GDFs. ATDC5 cells were cultured for $2 w$ in the absence (Fig.2A, 0, Fig.2B, Control) or in the presence four dose of GDF-5, GDF-6, or GDF-7. Representative alcian blue staining of ATDC5 culture in the absence or presence of each GDFs (A) and the intensity of staining measured by solubilized alcian blue stain using light absorbance at $600 \mathrm{~nm}(B) .{ }^{*} p<0.05$ when compared with control or each dose of GDFs.
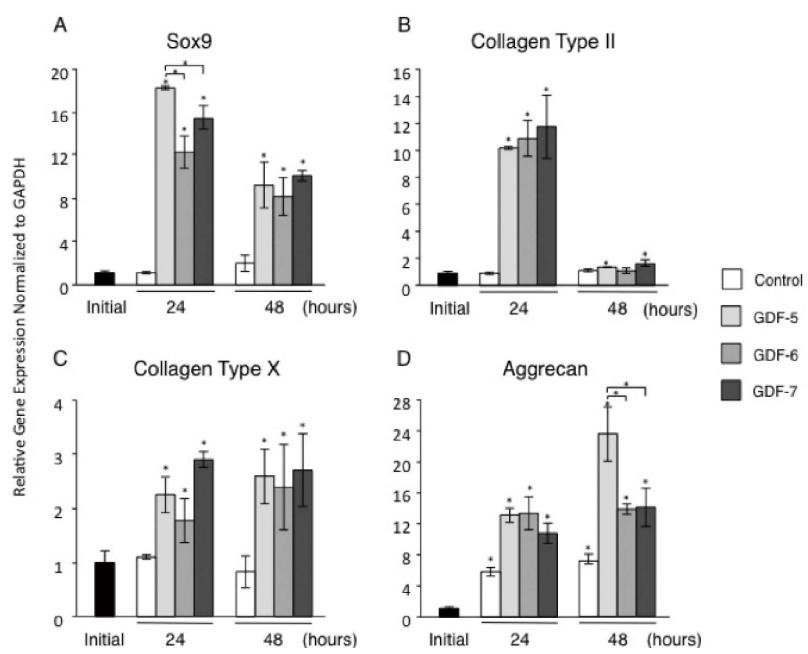

Figure 3. The gene expression profile of chondrogenic differentiation marker promoted by each GDFs. ATDC5 cells were cultured for 24 or 48 hours in the absence (Control) or presence (GDF-5, GDF-6, and GDF-7) of each GDFs at 50ng/ml. Relative gene expression was assayed by real-time RT-PCR and normalize to that of GAPDH. Each gene expression was designated as ratio to that at initial cell attachment as one (Initial). *p<0.05 when compared with initial or each GDFs.

\section{Discussion}

Previous studies have suggested that GDF-5, GDF-6, and GDF-7 all play important roles in chondrogenesis or chondrogenic cell differentiation in vitro and in vivo [1,3-5,7-9]. It remains unclear, however, whether these GDFs have different effects on chondrogenic cell differentiation or chondrogenic matrix production.

In our study, GDF-5, -6, and -7 induced cartilaginous 
nodule formation dose-dependently after two weeks of culture in the absence of insulin and ascorbic acid. The ATDC5 cell line was originally established from mouse embryonic teratocarcinoma cells which, when supplemented with $10 \mathrm{~g} / \mathrm{ml}$ insulin in maintenance medium containing sodium selenite and transferrin, could undergo sequential chondrogenic proteoglycan synthesis [12]. ATDC5 cells that were treated on day 7 of culture with ascorbic 2-phosphate along with insulin in DMEM/F12 produced larger and more abundant cartilaginous nodules on day 21 of culture than ATDC5 cells cultured in the absence of ascorbic 2-phosphate did [13]. However, a previous study reported that GDF-5 enhanced cartilage nodule formation in ATDC5 cells over three weeks (21days) of culture in DMEM/F12 differentiation medium containing ascorbic acid and $\beta$-glycerophosphate but not insulin [14].

Additionally, transforming growth factor beta-1 (TGF $\beta 1$ ) could promote chondrogenic differentiation as indicated by an increase in alcian blue staining intensity after 17 days of culture with alpha-modified Eagle Medium ( $\alpha$ MEM) in the absence of ascorbic acid, $\beta$-glycerophosphate, and insulin [15]. In human mesenchymal stromal cells derived from bone marrow and cultured in both a growth medium including insulin and an osteo-inductive medium including $\beta$-glycerophosphate, BMP-13 (GDF-6) promoted proteoglycan synthesis as indicated by alcian blue staining [6]. It has also been reported that GDF-7 increased alcian blue staining, suggesting extracellular matrix formation, in cells from the mouse pluripotent mesenchymal cell line C3H10T1/2 cultured in DMEM containing ascorbic acid and $\beta$-glycerophosphate [7]. Thus our result suggests that, in ATDC5 cells, GDF-5, -6 and -7 all promote the formation of cartilaginous nodules or the production of proteoglycans as indicated by alcian blue staining in a dose-dependent manner after two weeks of culture, even in the absence of other chondrogenesis-inducing factors such as ascorbic acid, $\beta$-glycerophosphate and insulin.

Our study demonstrated that all three of the exogenous GDFs that we studied promoted chondrogenic marker gene expressions, and in particular that, compared with GDF-6 and GDF-7, GDF-5 significantly promoted the gene expression of Sox 9 after 24 hours of culture and that of aggrecan after 48 hours culture. Sox9 is expressed predominantly in cells in mesenchymal condensations during the early skeletal development of embryos [16], and an overexpression of Sox 9 enhanced aggrecan expression in a mouse articular cartilage-derived cell line [17]. Thus our finding that exogenous GDF-5 increases the expression of aggrecan after 48 hours of culture may depend on the fact that it first increases the expression of Sox 9 after 24 hours of culture.

In ATDC5 cells, exogenous GDF-5 increased the expression of Col2 after one day of culture and further increased it after nine days of culture, as revealed by Northern Blot analysis [14]. In addition, exogenous GDF-5 increased aggrecan expression in periodontal ligament cells although it did not increase the expression of Sox9 [18]. It has been suggested that GDF-6 may promote the formation of chondrogenic gene products while inhibiting or retarding osteo-inductive signaling [6]. On the other hand, it has been reported that GDF-7 stimulates alcian blue staining, which is indicative of extracellular matrix formation, without affecting aggrecan gene expression in the mouse pluripotent mesenchymal cell line C3H10T1/2 [7]. Furthermore, cells of the MC615 chondrocyte cell line derived from the limbs of 14-day mouse embryos can simultaneously express chondrocytic and osteoblastic markers in response to BMP-2 or -4 , but show minimal response to BMP-12 (or GDF-7) or BMP-13 (or GDF-6) [19]. Taken together, our results indicate that, in the ATDC5 chondrogenic cell line, GDF-5, -6 and -7 can all promote chondrogenic cell differentiation marker genes, and especially that GDF-5 has a more potent effect than GDF-6 and GDF-7 have on the increase in aggrecan expression that is induced by Sox9. In summary, our study suggests that GDF-5, -6 and -7 do have functional differences in early chondrogenic cell differentiation with regard to chondrogenic gene expression. Our findings may be useful in the field of tissue regeneration as these GDFs could serve as molecular manipulators of cell differentiation.

\section{Acknowledgements}

We acknowledge sponsorship of this work by the Center for Regenerative Medicine, Fukuoka Dental College, MEXT-Supported Program for the Strategic Research Foundation at Private Universities (S1001059) from the Ministry of Education, Culture, Sports, Science and Technology of Japan. This work also has been supported by the Advanced Science Research Center and by Grants-in-Aid for Scientific Research (No.17791508) from the Ministry of Education, Culture, Sports, Science and Technology of Japan.

\section{References}

[1] Storm EE., Huynh TV., Copeland NG., Jenkins NA., Kingsley DM., Lee SJ. (1994) Limb alterations in brachypodism mice due to mutations in a new member of the TGF beta-superfamily. Nature. 368(6472):639-43.

[2] Sanna S., Jackson AU., Nagaraja R., Willer CJ., Chen WM., Bonnycastle LL., Shen H., Timpson N., Lettre G., Usala G., Chines PS., Stringham HM., Scott LJ., Dei M., Lai S., Albai G., Crisponi L., Naitza S., Doheny KF., Pugh EW., Ben-Shlomo Y., Ebrahim S., Lawlor DA., Bergman RN., Watanabe RM., Uda M., Tuomilehto J., Coresh J., Hirschhorn JN., Shuldiner AR., Schlessinger D., Collins FS., Davey Smith G., Boerwinkle E., Cao A., Boehnke M., Abecasis GR., Mohlke KL. (2008) Common variants in the GDF5-UQCC region are associated with variation in human height. Nat Genet. 40(2):198-203.

[3] Yoshimoto T., Yamamoto M., Kadomatsu H., Sakoda K., Yonamine Y., Izumi Y. (2006) Recombinant human growth/differentiation factor-5 (rhGDF-5) induced bone formation in murine calvariae. J Periodontal Res. 41(2):140-7. 
[4] Hatakeyama Y., Tuan RS., Shum L. (2004) Distinct functions of BMP4 and GDF5 in the regulation of chondrogenesis. J Cell Biochem. 91(6):1204-17.

[5] Erlacher L., Ng CK., Ullrich R., Krieger S., Luyten FP. (1998) Presence of cartilage-derived morphogenetic proteins in articular cartilage and enhancement of matrix replacement in vitro. Arthritis Rheum. 41(2):263-73.

[6] Shen B., Bhargav D., Wei A., Williams LA., Tao H., Ma DD., Diwan AD. (2009) BMP-13 emerges as a potential inhibitor of bone formation. Int J Biol Sci. 5(2):192-200

[7] Yeh LC., Lee JC. (2010) Effects of cartilage-derived morphogenetic protein-3 on the expression of chondrogenic and osteoblastic markers in the pluripotent mesenchymal C3H10T1/2 cell line. Growth Factors. 28(2):117-28.

[8] Mikic B., Ferreira MP., Battaglia TC., Hunziker EB. (2008) Accelerated hypertrophic chondrocyte kinetics in GDF-7 deficient murine tibial growth plates. J Orthop Res. 26(7):986-90.

[9] Gooch KJ, Blunk T, Courter DL, Sieminski AL, Vunjak-Novakovic G, Freed LE. (2002) Bone morphogenetic proteins-2, -12 , and -13 modulate in vitro development of engineered cartilage. Tissue Eng. 8(4):591-601.

[10] Hatakeyama Y., Hatakeyama J., Takahashi A., Oka K., Tsuruga E., Inai T., Sawa Y. (2011) The effect of valproic acid on mesenchymal pluripotent cell proliferation and differentiation in extracellular matrices. Drug Target Insights. 5:1-9.

[11] Nagata M., Nuckolls GH., Wang X., Shum L., Seki Y., Kawase T., Takahashi K., Nonaka K., Takahashi I., Noman AA., Suzuki K., Slavkin HC. (2011) The primary site of the acrocephalic feature in Apert Syndrome is a dwarf cranial base with accelerated chondrocytic differentiation due to aberrant activation of the FGFR2 signaling. Bone. 48(4):847-56.
[12] Atsumi T, Miwa Y, Kimata K, Ikawa Y. (1990) A chondrogenic cell line derived from a differentiating culture of AT805 teratocarcinoma cells. Cell Differ Dev. 30(2):109-16.

[13] Altaf FM., Hering TM., Kazmi NH., Yoo JU., Johnstone B. (2006) Ascorbate-enhanced chondrogenesis of ATDC5 cells Eur Cell Mater. 12:64-9

[14] Nakamura K, Shirai T, Morishita S, Uchida S, Saeki-Miura K, Makishima F. (1999) p38 mitogen-activated protein kinase functionally contributes to chondrogenesis induced by growth/differentiation factor-5 in ATDC5 cells. Exp Cell Res. 250(2):351-63.

[15] Han F., Adams CS., Tao Z., Williams CJ., Zaka R., Tuan RS., Norton PA., Hickok NJ. (2005) Transforming growth factor-beta1 (TGF-beta1) regulates ATDC5 chondrogenic differentiation and fibronectin isoform expression. Cell Biochem. 95(4):750-62.

[16] Wright E., Hargrave MR., Christiansen J., Cooper L., Kun J., Evans T., Gangadharan U., Greenfield A., Koopman P. (1995) The Sry-related gene Sox9 is expressed during chondrogenesis in mouse embryos. Nat Genet. 9(1):15-20.

[17] Sekiya I, Tsuji K, Koopman P, Watanabe H, Yamada Y, Shinomiya K, Nifuji A, Noda M. (2000) SOX9 enhances aggrecan gene promoter/enhancer activity and is up-regulated by retinoic acid in a cartilage-derived cell line, TC6. J Biol Chem. 275(15):10738-44.

[18] Xia D., Sumita Y., Liu Y., Tai Y., Wang J., Uehara M., Agata H., Kagami H., Fan Z., Asahina I., Wang S., Tran SD. (2013) GDFs promote tenogenic characteristics on human periodontal ligament-derived cells in culture at late passages. Growth Factors. 31(5):165-73.

[19] Valcourt U1, Ronzière MC, Winkler P, Rosen V, Herbage D, Mallein-Gerin F. (1999) Different effects of bone morphogenetic proteins $2,4,12$, and 13 on the expression of cartilage and bone markers in the MC615 chondrocyte cell line. Exp Cell Res. 251(2):264-74. 